\title{
Modificaciones de la amplitud articular de to- billo durante la marcha en niños afectos de PCI y pie equino tratados con TBA
}

\author{
O. BARCELO' ', I. GUIJARRO ${ }^{2}$, F. GARCÍA-MURO ${ }^{3}$, I. BUSTURIA ${ }^{4}$, P. GARCÍA ${ }^{5}$, I. PASCUAL-PASCUAL ${ }^{6}$, \\ L. GARCÍA-RAMA ${ }^{2}, F$. VERGARA \\ ${ }^{1}$ Departamento de Fundamentos de la Motricidad y del Entrenamiento Deportivo. \\ Facultad de Ciencias de la Actividad Física y del Deporte. UEM. \\ ${ }^{2}$ Departamento de Fisioterapia. Escuela de Fisioterapia.UEM \\ ${ }^{3}$ Fisioterapeuta. Ejercicio libre. \\ ${ }^{4}$ Departamento de Ciencias Morfológicas. Facultad de Ciencias de la Salud. \\ ${ }^{5}$ Unidad de Neurología. Fundación Jiménez Díaz \\ ${ }^{6}$ Unidad de Neuropediatría. Hospital la Paz \\ ${ }^{7}$ Unidad de Fisioterapia. Hospital de Móstoles
}

\begin{abstract}
Resumen
El objetivo de este estudio fue comprobar el efecto de la TBA (BOTOXâ) en la amplitud articular del tobillo durante la marcha en pacientes afectos de PCI. La muestra estuvo formada por 9 niños con una media de 6 años de edad distribuyéndose en 4 grupos de estudio: control, hemipléjico, dipléjico I y dipléjico II. Las variables cuantificadas fueron la máxima flexión dorsal y plantar realizadas durante la marcha (calzados y descalzos) así como los porcentajes del CM en el que se alcanzaron estos máximos. La técnica instrumental utilizada fue un sistema de fotogrametría vídeo $3 D$ con el software Kinescan-IBV. Interrelacionando las cuatro variables sometidas a estudio, los resultados obtenidos demostraron que cada grupo evolucionó de forma distinta. Mientras que el grupo hemipléjico alcanzó los mejores resultados entre los 3 meses y 5 meses dependiendo de la condición, el grupo dipléjico I lo consiguió a los 5 meses y 3 semanas el dipléjico II a los 9 meses y 3 semanas. La TBA permitió una mayor movilidad en la articulación del tobillo, sin embargo para que también mejorara la marcha fue necesario que transcurriera un cierto tiempo para reeducarla, necesitando un incremento de este periodo y del número de dosis de TBA cuanto mayor es la alteración de la marcha.
\end{abstract}

Palabras claves: biomecánica, parálisis cerebral infantil, toxina botulínica, marcha humana.

\section{Summary}

The purpose of this investigation was to analyse the effects of botulinum toxin (BT, from BOTOXâ) in the ankle range of motion during the walking gait of children with cerebral palsy $(C P)$ and foot in equinus. Nine children were selected as subjects and were assigned to each of the following groups: control, hemiplegic, diplegic, diplegic I and diplegic II. The main variables been analysed were: maximum dorsal and plantar flexion of the foot (both with bare and non-bare foot), and the \% of walking gait that elicited the maximum values of the dorsal and plantar flexion of the foot. A video three-dimensional system (Kinescan-IBV) was used for data analysis. The results demonstrated that each of the four groups showed a different response to the TB treatment. The hemiplegic group reached the best results between 3 and 5 months, whereas the diplegic I and diplegic II groups exhibited the best results at 5 months and 3 weeks, and at 9 months and 3 weeks, respectively. The $T B$ did increase the amplitude of the range of motion in the ankle joint, although a minimum period of time was required before these significant improvements were noted. In addition, this minimum period of time as well as the required dosage of TB were higher in those children showing greater alterations of the walking gait.

Keywords: biomechanics, cerebral palsy, botulinum toxin, walking gait. 


\section{Introducción}

Un alto porcentaje de personas afectadas con parálisis cerebral infantil (PCI) presenta una forma de cualidad espástica que puede manifestarse como tetraparesia, diplejía o hemiplejía. La espasticidad presente en distintos grupos musculares junto con el desequilibrio de sus antagonistas provoca una ausencia del control selectivo y fásico de la actividad muscular en el curso de la realización de un gesto, como puede ser la marcha, y un desequilibrio biomecánico cuyo resultado puede ser, entre otros, el acortamiento longitudinal del músculo restringiendo o impidiendo la movilidad articular (Renders y cols 1997).

Para mejorar las posibilidades funcionales en este tipo de afectación se han utilizado una gran variedad de fármacos tales como el diacepam o baclofen, actualmente en desuso por sus efectos secundarios sobre las funciones superiores. También se ha recurrido a las inyecciones intramusculares de fenol siendo su utilización muy limitada por su naturaleza destructiva. La rizotomía posterior selectiva junto con las bombas de baclofen intratecal se han empleado con éxito pero el riesgo que supone la utilización de anestesia general, riesgo de meningitis y monitorización han hecho que actualmente sea la toxina botulínica de tipo A(TBA) el fármaco más empleado en la lucha contra la espasticidad con mínimos o nulos efectos secundarios. (Brin 1995).

La TBA, fue utilizada por primera vez en Oftalmología para el tratamiento del estrabismo y del blefarospasmo (Scott 1980). A partir de los resultados obtenidos se extendió su aplicación al campo de la Neurología en el tratamiento de distonías faciales, tortícolis espasmódica, disfonía espástica y de la espasticidad (Jankovic y Schwartz 1990; Cole y cols 1995) siendo aceptada actualmente como tratamiento rutinario de estas alteraciones (Lees

\section{Correspondencia:}

Olga Barceló Guido, Dpto. de Fundamentos

de la Motricidad y del Entrenamiento Deportivo Facultad de Ciencias de la Actividad Física y del Deporte.

Universidad Europea-CEES

C/ Tajo s/n. Urbanización El Bosque.

Villaviciosa de Odón. Madrid 28670

Spain

E-mail: olga.barcelo@fis.cisa.uem.es
1992).

Son numerosos los estudios realizados refiriendo óptimos resultados con la utilización de la TBA en el tratamiento de los músculos espásticos en el adulto (Das y Park 1989; Dunne y cols 1995) así como en niños y adolescentes afectos de parálisis cerebral espástica (Cosgrove y cols 1994; Koman y cols 1994; García 1996) valorando su eficacia en la disminución del tono muscular y aumento de las posibilidades de estiramiento de los músculos afectos reduciéndose la instauración de contracturas rígidas y su repercusión en la marcha (Prat 1994).

Otros trabajos han analizado las modificaciones producidas en el pie equino, pie equino-varo y pie equino-valgo por espasticidad y desequilibrio muscular en niños con parálisis cerebral al inyectarse en los grupos musculares afectos TBA ( Dengler y cols 1992 ; Koman y cols 1994; Mousny y Allington 1999) concluyendo que si estas alteraciones morfológicas son reductibles, la TBA constituye una terapia coadyuvante que debe ser valorada y tenida en cuenta para conseguir una mejoría en su funcionalidad y potencial de deambulación.

Para valorar el grado de disminución de la espasticidad, el aumento de amplitudes articulares y la mejoría de las capacidades funcionales, en particular de la estación de pie y de la marcha, de los sujetos con parálisis cerebral tras la utilización de TBA se han seguido distintas metodologías de análisis como son: Physician Rating Scale (PRS) (Sánchez-Carpintero y Narbona 1997; Dunne y cols 1995), escala de evaluación global de O’Brien y de espasticidad de Ashwort (Pascual-Pascual y cols 1997), medición goniométrica de las amplitudes articulares y análisis videográfico (Mousny y Allington 1999), electromiografía de superficie (Renders y cols 1997).

Vista las preguntas que quedan sin resolver de este área de estudio, el objetivo de este trabajo de investigación fue no sólo las mejoras articulares del tobillo sino también la repercusión de dichas mejorías en el desarrollo del patrón de marcha.

\section{Materiales y métodos}

\section{Sujetos}

La muestra estuvo formada por 9 niños ( 3 varones y 6 féminas) de $5,1 \pm 1,8$ años (media $\pm \mathrm{DE}$ ) $20,3 \pm 3,1 \mathrm{~kg}$; dicha muestra se dividió en 4 grupos de estudio: grupo control o de referencia de individuos sanos $(n=3)(\mathrm{GC})$; grupo hemipléjico $(\mathrm{n}=3)$ $(\mathrm{GH})$, grupo dipléjico I $(\mathrm{n}=2)(\mathrm{GD}$ I) y grupo 
dipléjico II ( $\mathrm{n}=1)$ (GD II).

Los sujetos de estudio fueron seleccionados por un neurólogo y un neuropediatra utilizando los mismos criterios clínicos para la selección de los pacientes así como de los grupos musculares dónde debía administrarse la TBA (BOTOXâ). En todos los grupos de niños afectados se utilizó una dosis de $6 \mathrm{U} / \mathrm{Kg}$ de peso corporal, inyectando un punto en cada gemelo, interno y externo, de la pierna o piernas afectadas. Los sujetos del GH y GD I recibieron a lo largo de este estudio dos aplicaciones de TBA y al sujeto del GD II, por criterio médico, tres; por este motivo este sujeto fue excluido del GD I para mantener una muestra lo más homogénea posible en cada grupo de estudio.

\section{Mediciones}

Para determinar las modificaciones producidas en la marcha debidas al tratamiento con TBA se realizaron 5 mediciones a lo largo de 5 meses y 21 días en los GH y GD I y 7 mediciones en el GD II durante 9 meses y 21 días. En los dos primeros, las 5 mediciones se distribuyeron de la siguiente manera: la primera, denominada basal (B), se realizó una semana antes de la primera dosis de TBA, la segunda a los 21 días (3s), periodo en el que según Cosgrove y cols (1994) y Mousny y Allington (1999) se producía el máximo efecto de la TB; la tercera medición se realizó a los 3 meses $(3 \mathrm{~m})$, la siguiente a los 5 meses $(5 \mathrm{~m})$ (una semana antes de la segunda dosis de TBA) y la última medición a los 5 meses y 21 días (5m3s).

En el GD II, las mediciones se realizaron de la siguiente forma: basal (B), a los 21 días (3s) (después de la $1^{\mathrm{a}}$ dosis de TBA), a los 3 meses ( $\left.3 \mathrm{~m}\right)$, a los 3 meses y 3 semanas después de la $2^{\mathrm{a}}$ dosis $(3 \mathrm{~m} 3 \mathrm{~s})$, a los 5 meses $(5 \mathrm{~m})$, a los 9 meses $(9 \mathrm{~m})$ y la
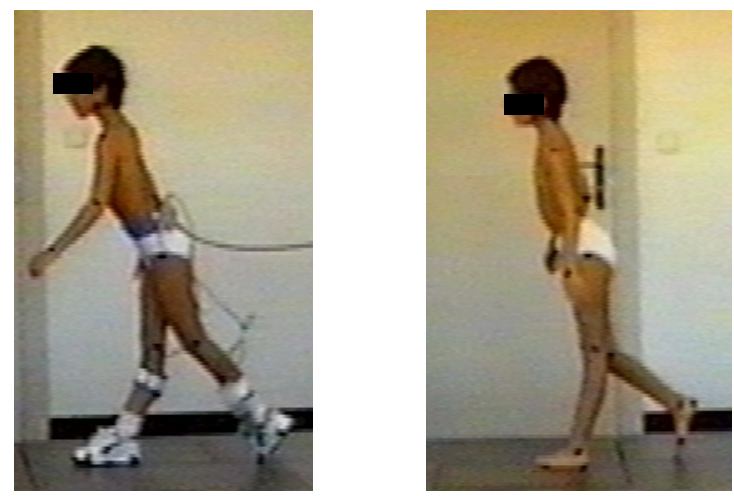

Figura 1. Paciente dipléjica con marcadores en los puntos anatómicos de interés en la condición calzada (a) y descalza (b). última a los 9 meses y 21 días ( 3 semanas después de la $3^{\mathrm{a}}$ dosis) $(9 \mathrm{~m} 3 \mathrm{~s})$. En todas las mediciones se evaluó a cada sujeto en dos condiciones: marcha calzado (Figura 1a) y marcha descalzo (Figura 1b), con el objetivo de eliminar el posible efecto ortesis que suele tener el calzado en sujetos con caída plantar por la espasticidad del tríceps sural. En cada medición el sujeto realizó un mínimo de 5 repeticiones con cada condición por un pasillo de marcha de $10 \mathrm{~m}$ de longitud a cadencia libre y se descartaron aquellas en las que la velocidad de la marcha variase significativamente.

\section{Selección de variables}

De acuerdo con los objetivos planteados para el estudio se cuantificaron cuatro variables cinemáticas:

- Máxima flexión dorsal de tobillo (FD): ángulo mayor de $90^{\circ}$ formado por el segmento pierna y el segmento pie proyectado sobre un plano sagital. Un aumento de este máximo indicaría una reducción del grado de Espasticidad de los gemelos.

- Máxima flexión plantar de tobillo (FP): ángulo menor de $90^{\circ}$ formado por el segmento pierna y el segmento pie proyectado sobre un plano sagital. Una disminución de esta variable indicaría una reducción del grado de equino.

En ambos casos se consideró la angulación de $90^{\circ}$ como la posición neutra (Figura 2 ).

Con estas dos variables se pudo comprobar la efectividad de la TBA en la amplitud articular del tobillo, pero para evidenciar si la evolución del patrón de la marcha se aproximaba o alejaba del patrón normal (PN) se consideró interesante cuantifi-

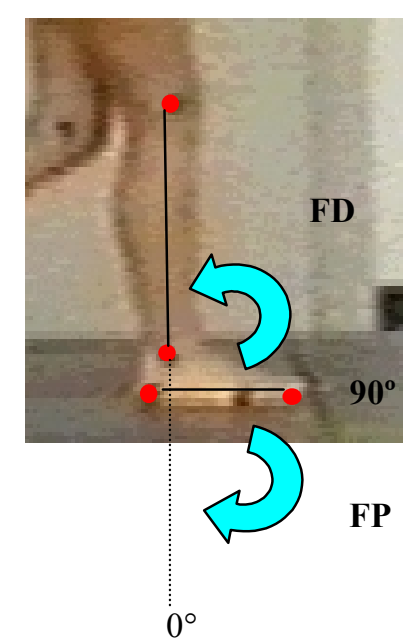

Figura 2. Modelo mecánico utilizado con las referencias angulares consideradas. 


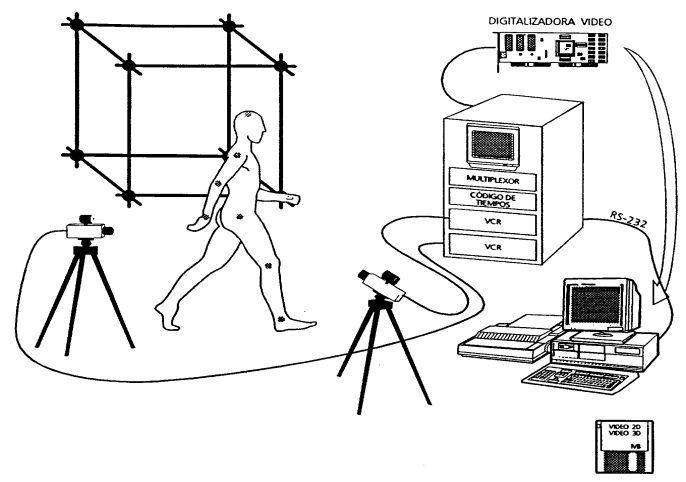

Figura 3. Esquema de funcionamiento del sistema de vídeo (Fuente: IBV).

car otras dos variables:

- Tiempo de la máxima flexión dorsal: este tiempo se transformó en porcentaje del ciclo de marcha (CM) considerando como el $100 \%$ a la duración total del ciclo o zancada (tiempo que transcurre entre dos apoyos consecutivos). Según Prat (1994), este máximo se realiza en el 48\% del CM correspondiendo al final de la fase de apoyo final (AF).

Tiempo de la máxima flexión plantar: Al igual que la variable anterior también se transformó en porcentaje del $\mathrm{CM}$, produciéndose en el $62 \%$, al principio de la fase de oscilación inicial (OI).

\section{Técnicas instrumentales}

Para este estudio se utilizaron dos técnicas instrumentales: un sistema de fotogrametría vídeo 3D y dos pares de células fotoelécticas.

El Sistema de Fotogrametría vídeo 3D (Figura 3) estuvo formado por dos cámaras Panasonic modelo AGDP800HE SVHS de vídeo sincronizadas entre sí y con una frecuencia de filmación de 50

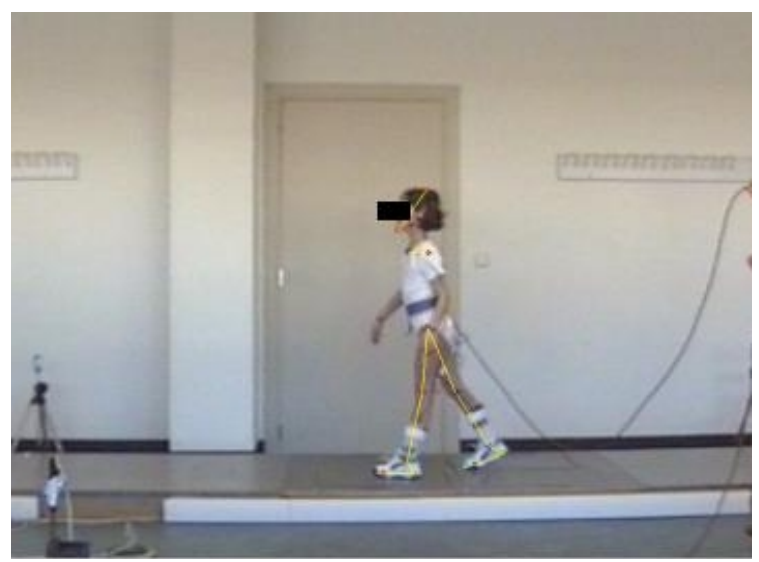

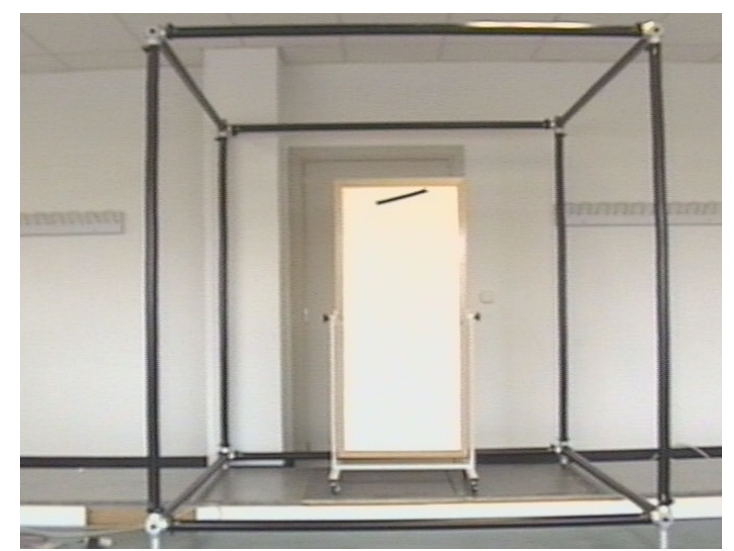

Figura 5. Sistema de referencia modular $2 \times 2$ (plano sagital).

fotogramas/segundo. Una de las cámaras se situó lateralmente al sujeto en un plano sagital (Figura 4a) y la otra frontalmente al mismo (Figura 4b), formando un ángulo de $90^{\circ}$, permitiendo el cálculo de las coordenadas tridimensionales por medio de una función de transformación lineal directa (DLT) (Abdel-Aziz y Karara 1971) del software KinescanIBV.

Para calibrar el espacio se utilizó un subsistema de referencia de 2 metros de lado (Figura 5). El error de digitalización se calculó digitalizando 10 veces un fotograma con el sistema de referencia modular, obteniéndose un error máximo de $0,05 \mathrm{~cm}$.

Previamente a la digitalización de las secuencias seleccionadas se definió un modelo mecánico formado por dos segmentos (pierna y pie) y cuatro marcadores (punta del pie, talón, tobillo y rodilla). Estos puntos fueron filtrados mediante splines de $5^{\circ}$ orden.

El sincronismo de los dos planos se realizó filmando dos LEDS, uno desde cada cámara, encendiéndose automáticamente al activarse las células

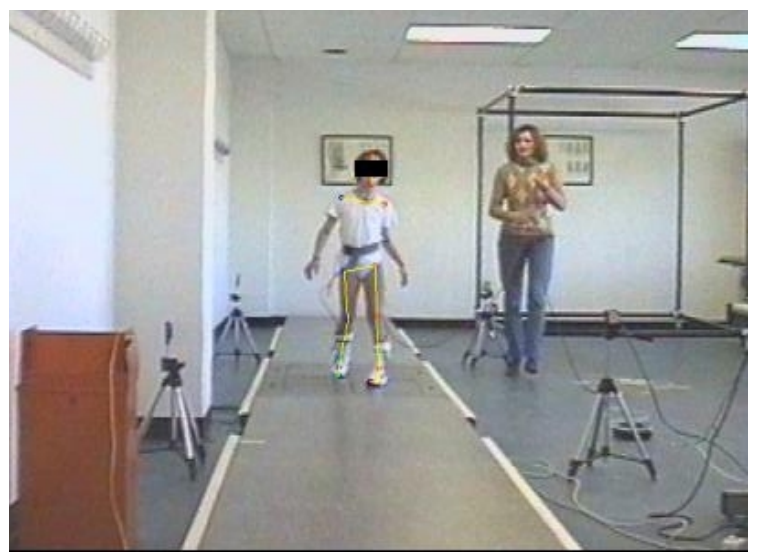

Figura 4. Planos de filmación. Plano sagital (a) y plano frontal (b). 
fotoeléctricas.

Con dos pares de células fotoeléctricas unidas a un cronómetro multitiempo se midió la velocidad de marcha. Esta variable sólo se utilizó como criterio de selección de los registros de marcha.

\section{Estadística}

Tras la exportación de los datos a hojas de cál- culo Excel 95 se trataron estadísticamente con el software SPSS 10.0 realizando un análisis multivariante mediante un modelo lineal general comprobando previamente el cumplimiento de las hipótesis del citado modelo y considerando las diferencias estadísticamente significativas con un valor $\mathrm{p}<0.05$.
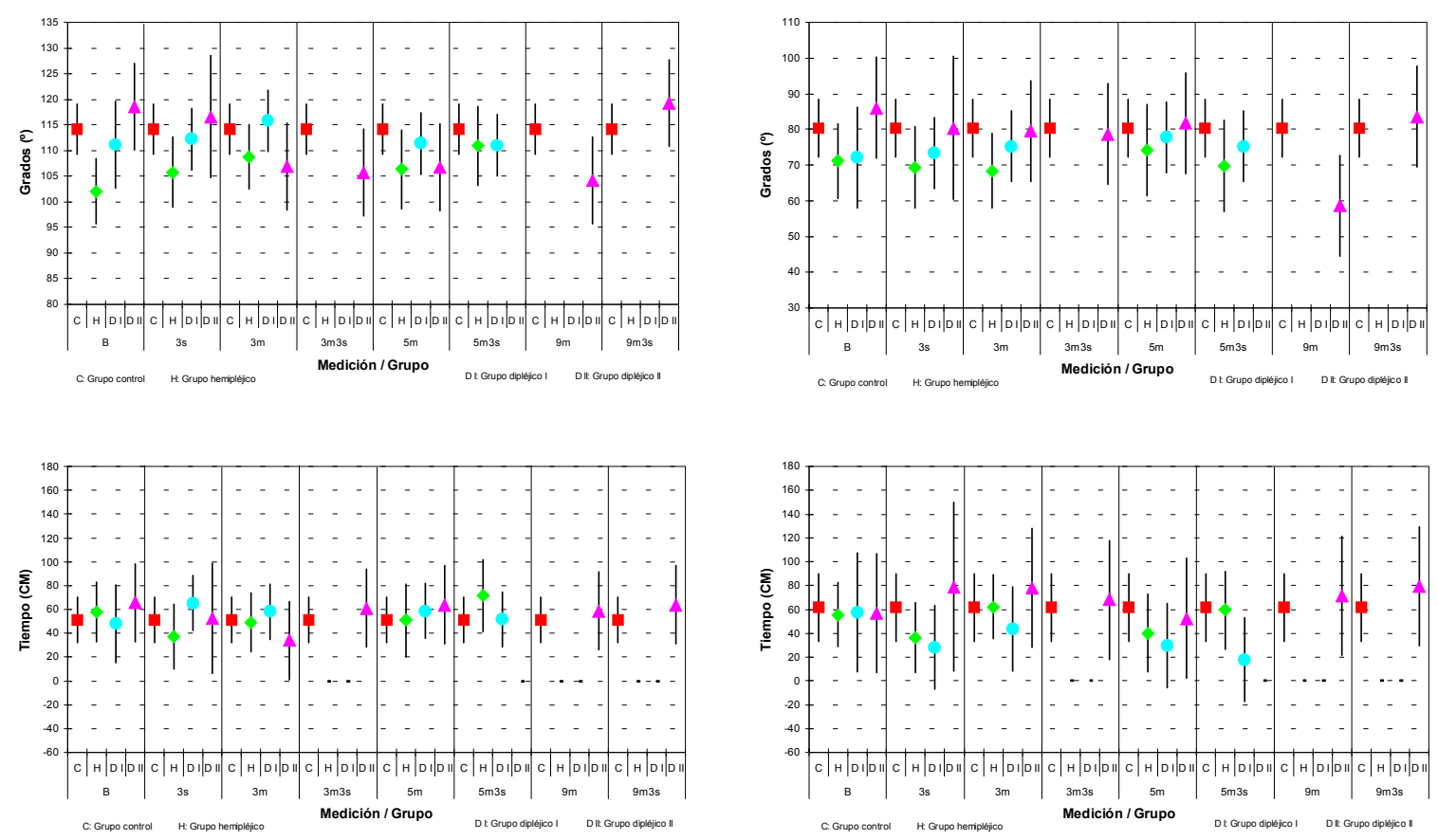

Figura 6. Comparación intragrupal e intergrupal de la: máxima FD (a), máxima FP (b), y sus respectivos tiempos (c y d) del tobillo derecho calzado a lo largo de las mediciones. (Límite superior, inferior y media)
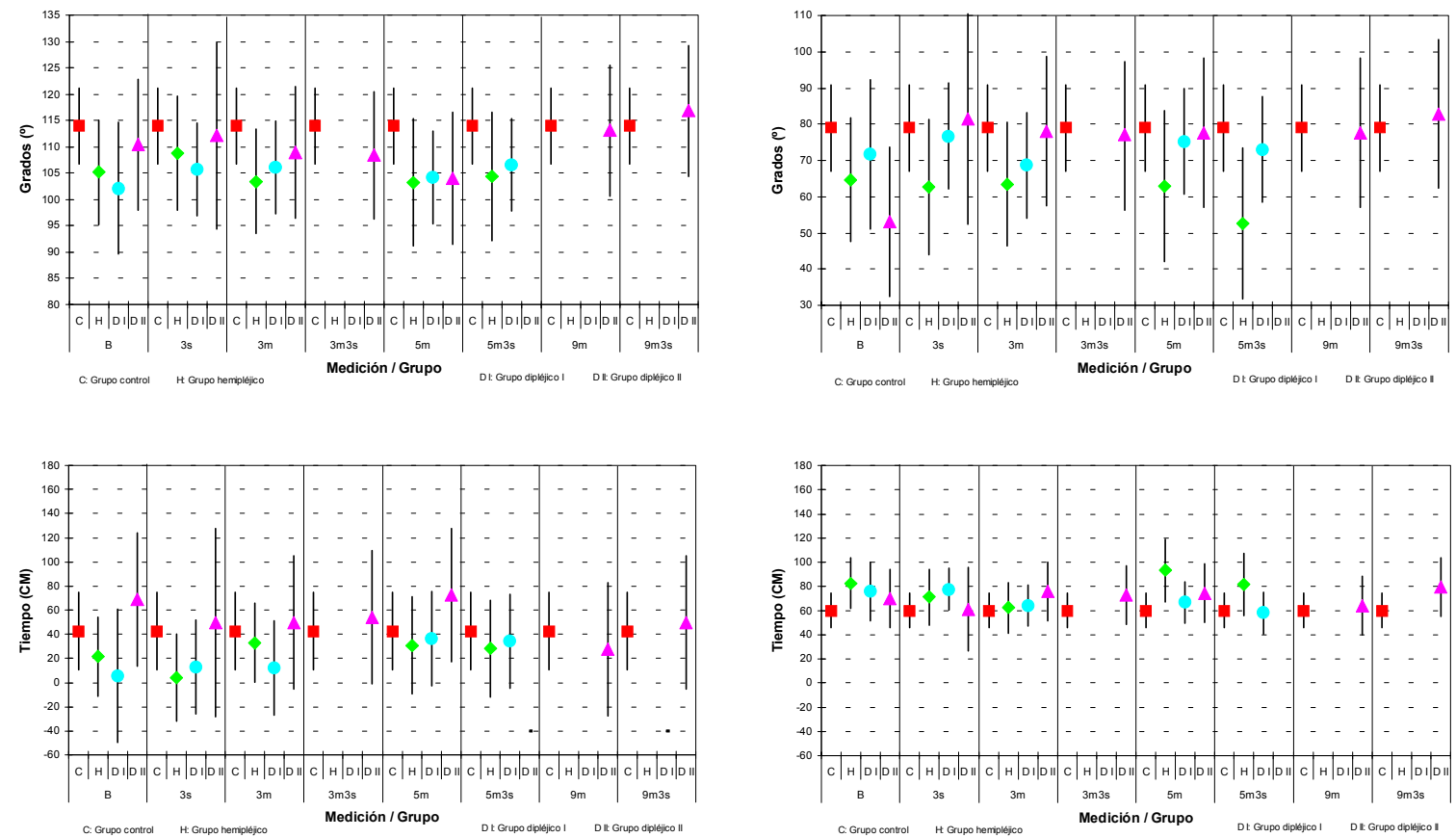

Figura 7. Comparación intragrupal e intergrupal de la: máxima FD (a), máxima FP (b), y sus respectivos tiempos (c y d) del tobillo izquierdo calzado a lo largo de las mediciones. (Límite superior, inferior y media) 


\section{Resultados y Discusión}

La discusión de los resultados se ha realizado diferenciando grupo clínico sometido a estudio, pie y condición utilizada.

\section{Grupo hemipléjico}

\section{Pie derecho condición calzado}

En el análisis de las variables cinemáticas se llegó a la conclusión de que si bien, la máxima FD se consiguió a los $5 \mathrm{~m} 3 \mathrm{~s}$ (Figura 6a) y el menor valor de la máxima FP a los $5 \mathrm{~m}$ (Figura 6b), interrelacionando las cuatro variables del estudio cinemático fue en la medición realizada a los $3 \mathrm{~m}$ cuando se produjo la mejoría más importante en este grupo clínico al registrarse en ella un aumento de la FD y una FP adecuada, siendo lo más importante el hecho de que estos máximos se realizaron en las fases del ciclo de la marcha descritas por la bibliografía para un sujeto sano (Figuras 6c y 6d), es decir, en el AF y principios de la OI respectivamente (Prat 1994).

En el estudio realizado por Cosgrove y cols (1994) en el que se evaluó la acción de la TBA sobre una población de 26 niños con PCI, se concluyó que tras la administración de TBA en el músculo tríceps sural se registró un aumento de la amplitud de FD del tobillo y disminución del grado de FP durante el ciclo de la marcha. Sin embargo, no se tuvo en cuenta la fase de la marcha en la que se registraron estos parámetros.

Si bien, por la acción bloqueante propia de la TBA que clínicamente da lugar a una parálisis flácida del músculo, las amplitudes articulares de FD aumentan por la activación de la musculatura flexora dorsal del tobillo, este hecho considerado de forma aislada podría no ser indicativo de la aproximación del niño afecto de parálisis espástica al patrón de marcha normal siendo necesario relacionar las amplitudes articulares conseguidas con las fases de la marcha en las que éstas aparecen.

\section{Pie izquierdo condición calzado}

Si se considerase, como se mencionó anteriormente, como único criterio de mejora el aumento aislado de la amplitud de la FD que se registró en el tobillo, se podría determinar que la mejoría en este grupo clínico se produjo a las 3s (Figura 7a) al ser en ésta dónde se registró la mayor angulación. Sin embargo, teniendo en cuenta además las otras variables del estudio cinemático, se pudo observar que en esta fase del estudio la máxima FD se produjo en el contacto (CI) (Figura 7c) pudiendo ser causado, por ejemplo, por una extensión inadecuada de rodilla, no considerándose entonces como una aproximación al patrón de marcha normal. Además, en esta medición la máxima FP (Figura 7b) se registró en la fase de oscilación media (OM) (Figura7d) pudiéndose producir un arrastre de los dedos e indicando de nuevo una alteración del patrón de marcha.

Por tanto, al analizar y relacionar las cuatro variables se pudo concluir que la mejoría se obtuvo a los $3 \mathrm{~m}$ puesto que, si bien la máxima FD fue ligeramente inferior a la obtenida a las $3 \mathrm{~s}$, ésta se registró en una fase de la marcha correcta (apoyo medio) y la FP, que presentó una angulación similar a la obtenida en la medición anterior, en la fase de despegue como se describe en el patrón de marcha normal.

\section{Pie derecho condición descalzo}

A los $5 \mathrm{~m}$ se alcanzó la máxima FD (cercana al GC) (Figura 8a) y el mínimo grado de FP (Figura $8 b)$ en un porcentaje del ciclo de la marcha adecuado. La FD se realizó en la fase de AF (Figura 8c) y la FP durante la preoscilación (OP) (Figura 8d) por lo que se consideró que fue en esta medición dónde se obtuvo la mayor aproximación al patrón de marcha de referencia

Cosgrove y cols (1994) observó que la máxima reacción muscular a la administración de TBA aparecía entre los 7 y 10 primeros días después de su aplicación y Mousny y Allington (1999) encontró el mayor incremento de los grados de FD pasiva de tobillo durante el primer mes. Si bien, la disminución de la espasticidad de los músculos posteriores de la pierna permitía conseguir un mayor estiramiento de éstos y por tanto una mayor amplitud de la FD de tobillo realizada pasivamente, no implicaba que los sujetos afectos de espasticidad muscular lo reprodujeran activamente en el mismo tiempo en el que la toxina presentaba sus mayores efectos sobre el músculo. Por ello cabe pensar, que si bien pasivamente los grupos musculares espásticos pudieran ser estirados con mayor facilidad, la utilización de esta nueva condición mecánica por parte del sujeto requería de más tiempo hasta conseguir el aprendizaje de patrones motores adecuados posibilitando la existencia de un mejor patrón de marcha aún cuando el efecto de la toxina sobre el músculo hubiera disminuido, como ocurrió en los resultados encontrados en este grupo, pie y condición después de cada inyección. 

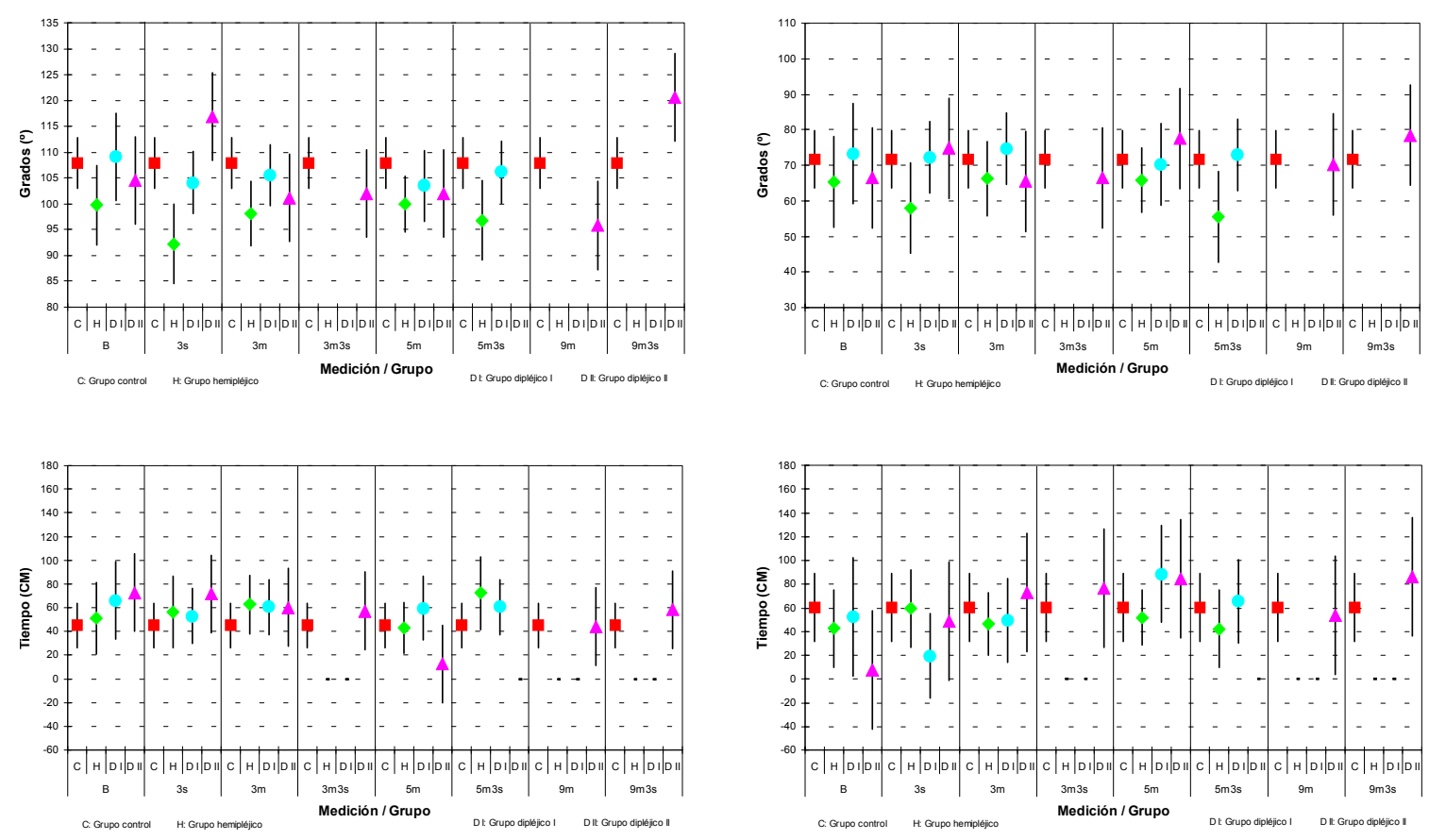

Figura 8. Comparación intragrupal e intergrupal de la: máxima FD (a), máxima FP (b), y sus respectivos tiempos (c y d) del tobillo derecho descalzo a lo largo de las mediciones. (Límite superior, inferior y media)

Pie izquierdo condición descalzo

Como menciona Prat (1994) en su libro, refiriéndose a la alteración que experimenta el patrón de marcha del miembro inferior no afecto en sujetos hemipléjicos, también se encontraron modificaciones en las variables de máxima FD, FP y porcentajes del $\mathrm{CM}$ de dicha extremidad.

En la medición $\mathrm{B}$ se obtuvieron la máxima FD (Figura 9a) y la mínima FP (Figura 9b), pero éstas se realizaron en fases incorrectas (Figuras 9c y 9d). Sin embargo, a los $5 \mathrm{~m}$ se produjo el siguiente valor mayor de FD y menor de FP, alcanzándose la primera en el AI y la segunda en la OI. Aunque la máxima FD se realizó precozmente, teniendo en cuenta los demás resultados obtenidos en las otras variables se consideró que fue en esta medición cuando el GH alcanzó la mayor mejoría.

\section{Grupo dipléjico $I$.}

Pie derecho condición calzado

Los resultados cinemáticos obtenidos dificultaron la determinación del momento del estudio en el que se produjo el acercamiento al patrón de referencia en este grupo clínico para este pie y condición. La única fase en la que el orden de consecución de las máximas angulaciones coincidió con el patrón de marcha normal fue en la medición $\mathrm{B}$ ( Figuras $6 \mathrm{c}$ y $6 \mathrm{~d}$ ) pero en ésta se registraron la mínima FD (Figura 6a) y el máximo grado de FP (Figura 6 b) por lo que no se podría considerar como el momento de mayor mejoría. A los $3 \mathrm{~m}$ se obtuvie- ron valores articulares adecuados pero en orden temporal inverso, que pudiera ser motivado por la brusca hiperextensión de rodilla que presentó uno de los sujetos de este grupo.

\section{Pie izquierdo condición calzado}

Al igual que sucedió en ambos pies con la condición descalza, la mejoría encontrada en el análisis videográfico apareció a los $5 \mathrm{~m} 3 \mathrm{~s}$ obteniéndose el mayor valor de FD (Figura 7a) de tobillo registrado a lo largo del estudio y una adecuada FP (Figura $7 b)$ realizadas en las fases de la marcha características de un PN (Figuras 7c y 7d).

\section{Pie derecho condición descalzo}

En este grupo clínico no aparecieron modificaciones importantes en los grados registrados de máxima FD (Figura 8a) y máxima FP (Figura 8b) en las distintas mediciones del estudio por lo que esta variable no pudo ser utilizada para determinar en qué fase se hubiera producido la posible mejora. No obstante, en el análisis del tiempo en el que se han realizado estos máximos se observó que se obtuvieron en el orden correcto en la medición realizada a los $5 \mathrm{~m} 3 \mathrm{~s}$ (Figuras $8 \mathrm{c}$ y $8 \mathrm{~d}$ ).

\section{Pie izquierdo condición descalzo}

Interrelacionando las cuatro variables sometidas a estudio se observaron mejorías en la medición 

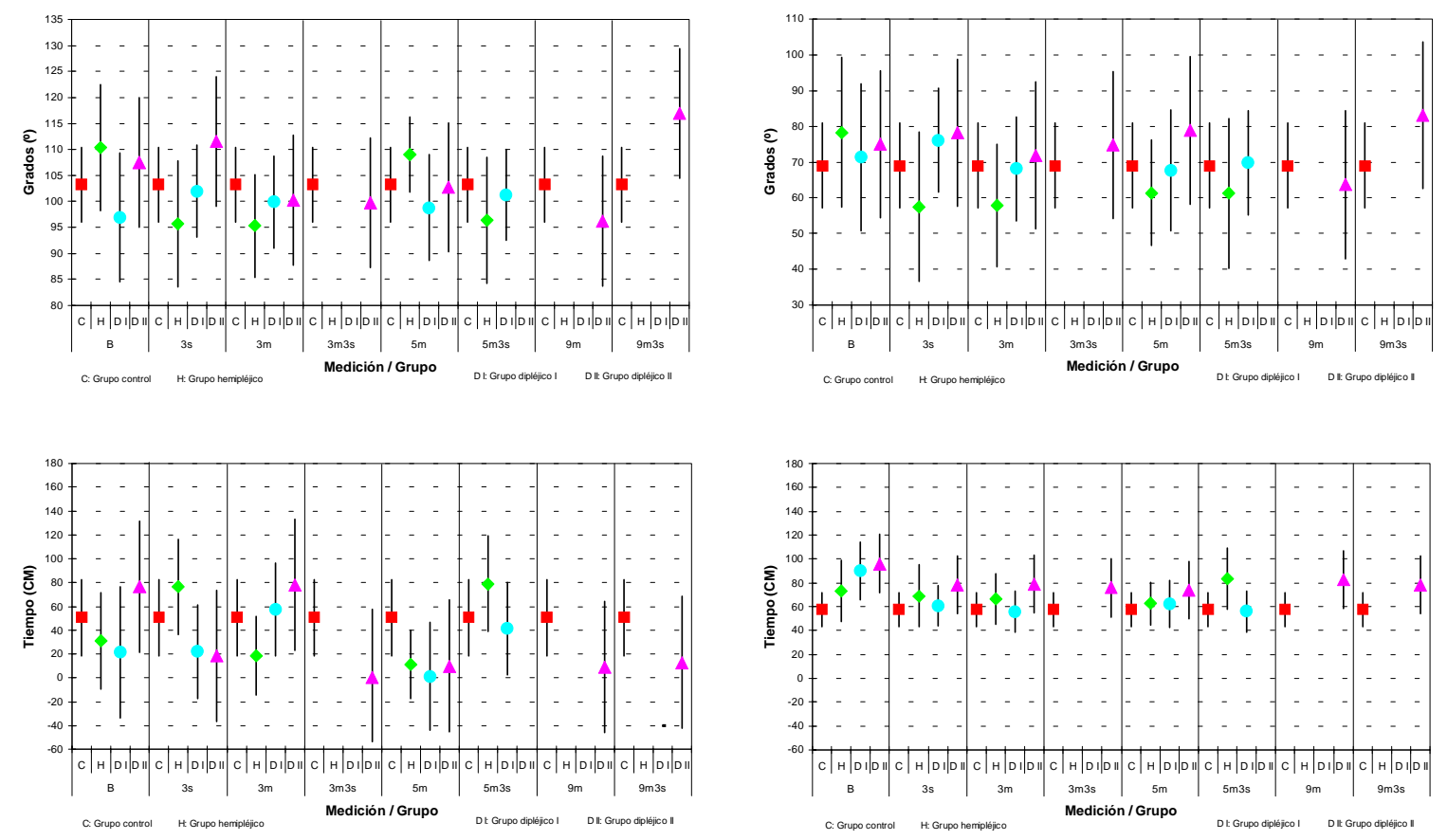

Figura 9. Comparación intragrupal e intergrupal de la: máxima FD (a), máxima FP (b), y sus respectivos tiempos (c y d) del tobillo izquierdo descalzo a lo largo de las mediciones. (Límite superior, inferior y media)

$3 \mathrm{~m}$ y $5 \mathrm{~m} 3 \mathrm{~s}$. En ambas mediciones los grados de FD (Figura 9a) y FP (Figura 9b) presentaron escasas diferencias pero fue en la última medición dónde las máximas angulaciones se alcanzaron en una secuencia temporal correcta (Figuras 9c y 9d).

\section{Grupo dipléjico II.}

Pie derecho condición calzado

Los valores angulares que más se acercaron al PN correspondieron a la medición 3s (Figuras 6a y $6 \mathrm{~b})$, justamente después de la primera dosis de TBA, realizándose la FD en el AF (Figura 6c) pero la FP en la OM (Figura 6d), pudiendo existir un posible arrastre de los dedos si no se utilizaran mecanismos de sustitución. A los $9 \mathrm{~m} 3 \mathrm{~s}$ se obtuvieron la máxima FD y la mínima FP realizadas en el orden secuencial correcto pero desfasadas ligeramente en el tiempo (en OI y OM respectivamente). Sin embargo, teniendo en cuenta los informes parciales realizados a lo largo del estudio de este grupo clínico, la medición $9 \mathrm{~m} 3 \mathrm{~s}$ se caracterizó por un mayor apoyo de retropié y mayor equilibrio aumentando la duración de la fase de apoyo y produciéndose, por tanto, este desfase. Por ello se consideró esta última fase como la de mayor mejoría.

\section{Pie izquierdo condición calzado}

Al igual que el pie derecho calzado las mejorías fueron encontradas a las $3 \mathrm{~s} \mathrm{y} 9 \mathrm{~m} 3 \mathrm{~s}$, pero a diferencia de éste también se obtuvieron indicios de mejoría en la penúltima medición. A las 3 semanas se consiguieron los valores angulares más cercanos al GC en la secuencia temporal correcta. El comportamiento a los 9m es similar al de las 3s a excepción de que en la primera la FD fue realizada precozmente, posiblemente por una extensión inadecuada de rodilla. En la medición $9 \mathrm{~m} 3 \mathrm{~s}$, al igual que en el pie derecho, se produce la máxima FD (Figura 7a) y la mínima FP (Figura 7b) realizándose ambas ligeramente posteriores al GC (Figuras 7 c y 7d), pero teniendo en cuenta otra vez lo expuesto con anterioridad en el pie derecho, podría corresponder a las fases correctas de una fase de apoyo incrementada.

\section{Pie derecho condición descalzo}

Aunque la FD más cercana al grupo de referencia se produjo en la medición $\mathrm{B}$, los máximos valores de FD fueron registrados a las $3 \mathrm{~s}$ y a los 9m3s (Figura 8a) coincidiendo con mínimas FP (como en el pie derecho e izquierdo en condición calzada) (Figura 8b). Pero es necesario indicar que con esta condición los valores angulares registrados a las 3s aparecieron invertidos en el tiempo (Figuras 8c y 8d), pudiendo ser representativo de un despegue precoz de talón. Sin embargo, los por 
centajes del CM en los que se han conseguido los valores angulares registrados a $10 \mathrm{~s} 9 \mathrm{~m} 3 \mathrm{~s}$ coinciden con el pie izquierdo calzado, por lo que también en esta condición se considera la mejoría en esta fase. Esta diferencia encontrada entre las mediciones $3 \mathrm{~s}$ y $9 \mathrm{~m} 3 \mathrm{~s}$ pudiera ser originada por la ausencia del efecto ortesis que proporcionaba el calzado por lo que se necesitaría más tiempo para que este grupo clínico consiguiera un mayor control sobre los movimientos que debe realizar el tobillo a lo largo del ciclo de la marcha.

\section{Pie izquierdo condición descalzo}

En este pie y condición se volvieron a registrar máximas FD (Figura 9a) así como mínimas FP (Figura $9 \mathrm{~b}$ ) a las $3 \mathrm{~s}$ y $9 \mathrm{~m} 3 \mathrm{~s}$, pero en ambas mediciones la FD se realizó precozmente en el AM (Figura 9c) mientras que la FP se produjo en la OM (Figura 9d).

En esta situación y a diferencia de las otras, la medición $5 \mathrm{~m}$ presentó una FD más cercana al GC pero se realizó más precozmente (AI) que a las $3 \mathrm{~s}$ y a los $9 \mathrm{~m} 3 \mathrm{~s}$, así como una FP similar a la que se obtuvo en la medición $3 \mathrm{~s}$ ejecutándose en la fase de OM, ligeramente más próxima al valor de referencia que en el resto de las mediciones.

\section{Conclusiones}

La TBA permitió una mayor movilidad en la articulación del tobillo, al reducir la espasticidad de los músculos gemelos tratados.

Aunque la TBA mejora la amplitud articular, esto no implica una mejora de la marcha si no se realiza en las fases adecuadas $(\% \mathrm{CM})$, no obteniéndose por tanto inmediatamente después de las dosis de TBA, sino posteriormente, ya que el sujeto necesita un cierto periodo de tiempo para integrar la nueva condición mecánica.

En el análisis y comparación de las cuatro variables cinemáticas en cada grupo clínico de estudio se llegó a la conclusión de que cuanto mayor fuera la alteración del patrón de marcha eran necesarias más dosis de TBA y más tiempo debía transcurrir hasta alcanzar unos valores próximos o iguales al GC.

\section{Agradecimientos}

Los autores desean agradecer a la empresa a Allergan S.A. por su financiación; al departamento de Tecnologías de la Comunicación de la Universi- dad Europea CEES por su apoyo tecnológico, especialmente a Moisés Rodríguez; a Joan Válios por su apoyo técnico y humano; a Susana Aznar por sus imprescindibles gestiones, a Alejandro Lucía por su orientación y a todos los que han colaborado desinteresamente ofreciéndonos en todo momento su ayuda (Alfonso Montero, Enrique Musumeci, Carolina Walker, Patricia Serrano, Enrique Navarro, Yves Sanvoisin) y en especial a todos los niños y padres que hicieron posible esta investigación.

\section{Bibliografía}

1. Abdel-Aziz YI, Karara HM. Direct linear transformation from comparator coordinates into object space coordinates in close-range photogrammetry. ASP Symposium on Close Range Photogrammetry. 1971

2. Brin MF. Treatment of spasticity using local injections of botulinum toxin. The Mount Sinai

Medical Center Demonstrations Skills Workshop Series. New York. USA. Enero 1995.

3. Cole R, Hallet M, Cohen LG. Double-blind trial of botulinum toxin for treatment of local hand dystonia. Mov Disord 1995; 10: 466-67

4. Cosgrove AP, Corry IS, Graham HK. Botulinum toxin in the management of the lower limb in cerebral palsy. Dev Med Child Neurol 1994; 36: 386-96.

5. Das TK, Park TM. Botulinum toxin in treating spasticity. Br J Clin Pract 1989; 43: 401-3

6. Dengler R, Neyer U, Wohlfarth K, Betting U, Janzik HH. Local botulinum toxin in the treatment of spastic drop foot. J Neurol 1992; 239:375-8.

7. Dunne JW, Heye N, Dunne SL. Treatment of chronic limb spasticity with botulinum toxin A. J Neurol Neurosurg Psychiatry 1995; 58:232-5.

8. García PJ. Botulinum A toxins in the treatment spasticity in cerebral palsy during childhood. Neurol 1996; 11:34-6.

9. Jankovic J, Schwartz K. Botulinum toxin injections for cervical dystonia. Neurol 1990; 40: 277-80.

10. Koman LA, Mooney III JF, Smith BP, Goodman A, Mulvaney T. Management of spasticity in cerebral palsy with botulinum-A toxin: report of a preliminary, randomized, double-blind trial. J pediatr orthop 1994; 14:299-303.

11. Lees AJ. Botulinum toxin. Br Med J 1992; 305: 116970.

12. Mousny M, Allington N. La toxine botulinique A dans le traitement de la spasticité dynamique en équin chez les enfants porteur d'infirmité motrice d'origine cérébrale. Rev Chir Orthop 1999; 85:15663.

13. Pascual-Pascual SI, Sánchez de Muniain P, Roche MC, Pascual-castroviejo I. La toxina Botulínica 
como tratamiento de parálisis cerebral infantil. Rev neurol 1997. 25:1369-75.

14. Prat J. Curso de Formación de Técnicos Ortoprotésicos Euroforum. Miembro Inferior y Marcha Humana. Valencia: Instituto de Biomecánica de Valencia. 1994.

15. Renders A, Detembleur C, Rossillon R, Lejeune TH, Rombouts JJ. L'apport de l'analyse électromyographique de la marche dans l'étude du pied spastique de l'infirme moteur d'origine cérébrale: étude préliminaire. Rev Chir Orthop 1997; 83:259-64.

16. Sánchez-Carpintero R, Narbona J. Toxina botulínica en parálisis cerebral infantil: resultados en 27 sujetos a lo largo de un año. Rev neurol 1997; 25:531-5.

17. Scott AB. Botulinum toxin injection into extra-ocular muscles as an alternative to strabismus surgery. Ophtalmol 1980; 87:1044-49. 